\title{
A Priori Estimation of the Solution for Mixed Problems with Integral Condition for Singular Parabolic Equations
}

\author{
Raid Almomani \\ Math Department, University of Sharjah, P.O. Box 27272, Sharjah, UAE \\ Correspondence should be addressed to Raid Almomani; raid@yu.edu.jo
}

Received 31 October 2013; Accepted 15 December 2013; Published 4 February 2014

Academic Editors: M. Escobedo and C. Zhu

Copyright () 2014 Raid Almomani. This is an open access article distributed under the Creative Commons Attribution License, which permits unrestricted use, distribution, and reproduction in any medium, provided the original work is properly cited.

We prove a theorem in which we get a priori estimation of the solution for mixed problems with integral condition for singular parabolic equations. Mixed problems with nonlocal boundary conditions or with nonlocal initial conditions were studied in many works lately. Our result plays an important role in the theory of heat transmission, thermoelasticity, chemical engineering, underground water flow, and plasma physics.

\section{Introduction}

The importance of problems with integral condition has been pointed out by Samarskii [1]. Mathematical modelling by evolution problems with a nonlocal constraint of the form $(1 /(1-\alpha)) \int_{\alpha}^{1} u(x, t) d x=E(t)$ is encountered in heat transmission theory, thermoelasticity, chemical engineering, underground water flow, and plasma physics. For background information, we refer the reader to Benouar and Yurchuk [2], Bouziani and Benouar [3], Bouziani [4], Cannon and van der Hoek [5-7] Ionkin and Moiceev [8, 9], Kamynin [10], and Yurchuk [11, 12]. Mixed problems with nonlocal boundary conditions or with nonlocal initial conditions were studied in Bouziani [13], Byszewski [14-16], Gasymov [17], Ionkin $[8,9]$, Lazhar [18], and Said and Nadia [19]. The results and the method used here are a further elaboration of those in [2]. We should mention here that the presence of integral term in the boundary condition can greatly complicate the application of standard functional and numerical techniques. This work can be considered as a continuation of the results in [11,20].

We consider the following mixed problem in the rectangle $\mathrm{Q}=(0, l) \times(0, T)$ :

$$
L u=\frac{\partial u}{\partial t}-\frac{1}{x^{m}} \frac{\partial}{\partial x}\left(x^{m} \frac{\partial u}{\partial x}\right)=f(x, t), \quad m>0,
$$

$$
\begin{gathered}
l u=u(x, 0)=\varphi(x), \quad|u(0, t)|<\infty, \\
\int_{\alpha}^{l} x^{m} u(x, t) d x=0, \quad \alpha>0 .
\end{gathered}
$$

\section{A Priori Estimate}

Let $E$ be the Hilbert space of all sufficiently smooth functions $u$ satisfying the second and third conditions in (2) and equipped with the norm

$$
\begin{aligned}
& \|u\|_{E}^{2} \\
& =\int_{Q}(l-x)\left[x^{m}\left|\frac{\partial u}{\partial t}\right|^{2}+\frac{1}{x^{m}}\left|\frac{\partial}{\partial x}\left(x^{m} \frac{\partial u}{\partial x}\right)\right|^{2} d x d t\right] \\
& \quad+\sup _{0 \leq t \leq T}\left\{\int_{0}^{l}(l-x) x^{m}\left(\frac{\partial x}{\partial u}\right)^{2} d x+\frac{m}{l-\alpha} \int_{\alpha}^{l} x^{m-1} u^{2} d x\right\} .
\end{aligned}
$$

The equality

$$
x^{m+1} \frac{\partial u}{\partial x}=\int_{0}^{x} d \eta \iint_{\eta}^{x} \frac{\partial}{\partial \xi}\left(\xi^{m} \frac{\partial u}{\partial \xi}\right) d \xi+\int_{0}^{x} \xi^{m} \frac{\partial u}{\partial \xi} d \xi
$$


implies the following inequality:

$$
\begin{aligned}
x^{m}\left|\frac{\partial u}{\partial x}\right| \leq & x^{1 / 2} \sqrt{\int_{0}^{x}\left(\frac{\partial}{\partial \xi}\left(\xi^{m} \frac{\partial u}{\partial \xi}\right)\right)^{2} d \xi} \\
& +\sqrt{\int_{0}^{x}\left(\xi^{m} \frac{\partial u}{\partial \xi}\right)^{2} d \xi}
\end{aligned}
$$

By (5) it follows that

$$
\lim _{x \rightarrow 0} x^{m} \frac{\partial u}{\partial x}=0
$$

for any $u \in E$.

We will use (5) for the solutions $u$ of the problem (1)-(2). For the right hand side $f$ of (1) and initial condition of $u$ from (2) we introduce the space $F$ which is consisted of the vector function $\mathscr{F}=(f, \varphi)$ with the norm

$$
\begin{aligned}
\|\mathscr{F}\|_{F}^{2}= & \int_{Q} x^{m}|f(x, t)|^{2} d x+\int_{0}^{l} x^{m}\left(\frac{\partial \varphi}{\partial x}\right)^{2} d x \\
& +\frac{m}{l-\alpha} \int_{\alpha}^{l} x^{m-1} \varphi^{2} d x .
\end{aligned}
$$

Here it is assumed that

$$
|\varphi(0)|<\infty, \quad \int_{\alpha}^{l} x^{m} \varphi(x) d x=0 .
$$

Theorem 1. For any function $u \in E$ such that $x^{m / 2}(\partial u / \partial t) \epsilon$ $L_{2}(Q)$ and $x^{-m / 2}(\partial / \partial x) x^{m}(\partial u / \partial x) \in L_{2}(Q)$, the following inequality holds:

$$
\|u\|_{E}^{2} \leq c\|\mathscr{F}\|_{F}^{2}
$$

where $c=2\left(l+\exp \left(T / 2 \alpha^{2}\right)\right)$.

Proof. We set $M u=x^{m} u$ with $0 \leq x \leq \alpha$ and

$$
\begin{gathered}
M u=\frac{l-x}{l-\alpha} x^{m} u(x, t)+\frac{1}{l-\alpha} J\left(x^{m} u\right), \\
J \vartheta(x)=\int_{\alpha}^{x} \vartheta(\xi) d \xi
\end{gathered}
$$

with $\alpha \leq x \leq l$.

Consider the following equality:

$$
\begin{aligned}
& \int_{0}^{\tau} \int_{0}^{l} L u M \frac{\partial u}{\partial t} d x d t \\
& \quad=\int_{0}^{\tau} \int_{0}^{l} \frac{\partial u}{\partial t} M \frac{\partial u}{\partial t} d x d t \\
& \quad-\int_{0}^{\tau} \int_{0}^{l} \frac{1}{x^{m}} \frac{\partial}{\partial x}\left(x^{m} \frac{\partial u}{\partial x}\right) M \frac{\partial u}{\partial t} d x d t .
\end{aligned}
$$

It can be seen that the following equalities hold:

$$
\begin{aligned}
& \int_{0}^{\tau} \int_{0}^{l} \frac{\partial u}{\partial t} M \frac{\partial u}{\partial t} d x d t \\
& =\int_{0}^{\tau} \int_{0}^{\alpha} x^{m}\left|\frac{\partial u}{\partial t}\right|^{2} d x \\
& +\int_{0}^{\tau} \int_{\alpha}^{l}\left[\left(\frac{l-x}{l-\alpha}\right) x^{m}\left|\frac{\partial u}{\partial t}\right|^{2}\right. \\
& \left.\quad+\frac{1}{l-\alpha} \frac{\partial u}{\partial t} \int_{\alpha}^{x} \frac{\xi \partial u(\xi, t) d \xi}{\partial t} d x d t\right]
\end{aligned}
$$

$$
\begin{aligned}
& \int_{\alpha}^{l} \frac{\partial u}{\partial t} \int_{\alpha}^{x} \xi^{m} \frac{\partial u(\xi, t)}{\partial t} d \xi d x \\
& \quad=\frac{m}{2} \int_{\alpha}^{l} \frac{\partial u}{\partial t}\left|\int_{\alpha}^{x} \xi^{m} \frac{\partial u(\xi, t)}{\partial t} d \xi\right|^{2} d x
\end{aligned}
$$

By (12), we obtain the following equality:

$$
\begin{aligned}
& \int_{0}^{\tau} \int_{0}^{l} \frac{\partial u}{\partial t} M \frac{\partial u}{\partial t} d x d t \\
& \quad=\int_{0}^{\tau} \int_{0}^{\alpha} x^{m}\left|\frac{\partial u}{\partial t}\right|^{2} d x d t \\
& \quad+\int_{0}^{\tau} \int_{\alpha}^{l} \frac{l-x}{l-\alpha} x^{m}\left|\frac{\partial u}{\partial t}\right|^{2} d x d t \\
& \quad+\frac{m}{l-\alpha} \int_{0}^{\tau} \int_{\alpha}^{l} \frac{1}{x^{m+1}}\left|\int_{\alpha}^{x} \xi^{m} \frac{\partial u(\xi, t)}{\partial t} d \xi\right|^{2} d x d t
\end{aligned}
$$

Integrating by parts (and using (6)), we get

$$
\begin{aligned}
& -\int_{0}^{\tau} \int_{0}^{\alpha} \frac{1}{x^{m}} \frac{\partial}{\partial x}\left(x^{m} \frac{\partial u}{\partial x}\right) x^{m} \frac{\partial u}{\partial t} d x d t \\
& =-\left.\int_{0}^{\tau} x^{m} \frac{\partial u}{\partial x} \frac{\partial u}{\partial t} d t\right|_{x=0} ^{x=\alpha}+\int_{0}^{\tau} \int_{0}^{\alpha} x^{m} \frac{\partial u}{\partial x} \frac{\partial^{2} u}{\partial x \partial t} d x d t \\
& =-\int_{0}^{\tau} \alpha^{m} \frac{\partial u(\alpha, t)}{\partial \alpha} \frac{\partial u(\alpha, t)}{\partial t} d t \\
& \quad+\frac{1}{2} \int_{0}^{\alpha} x^{m}\left(\frac{\partial u(x, \tau)}{\partial x}\right)^{2} d x-\frac{1}{2} \int_{0}^{\alpha} x^{m}\left(\frac{d \varphi(x)}{d x}\right)^{2} d x
\end{aligned}
$$




$$
\begin{aligned}
& -\int_{0}^{\tau} \int_{\alpha}^{l} \frac{l-x}{l-\alpha} \frac{1}{x^{m}} \frac{\partial}{\partial x}\left(x^{m} \frac{\partial u}{\partial x}\right) x^{m} \frac{\partial u}{\partial t} d x d t \\
& =-\left.\int_{0}^{\tau} \frac{l-x}{l-\alpha} x^{m} \frac{\partial u}{\partial x} \frac{\partial u}{\partial t} d t\right|_{x=\alpha} ^{x=l} \\
& +\int_{0}^{\tau} \int_{\alpha}^{l} \frac{l-x}{l-\alpha} x^{m} \frac{\partial u}{\partial x} \frac{\partial^{2} u}{\partial x \partial t} d x d t \\
& -\frac{1}{l-\alpha} \int_{0}^{\tau} \int_{\alpha}^{l} x^{m} \frac{\partial u}{\partial x} \frac{\partial u}{\partial t} d x d t \\
& =\int_{0}^{\tau} \alpha \frac{\partial u(\alpha, t)}{\partial \alpha} \frac{\partial u(\alpha, t)}{\partial t} d t \\
& +\frac{1}{2} \int_{\alpha}^{l} \frac{l-x}{l-\alpha} x^{m}\left(\frac{\partial u(x, \tau)}{\partial x}\right)^{2} d x \\
& -\frac{1}{2} \int_{\alpha}^{l} \frac{l-x}{l-\alpha} x^{m}\left|\frac{\partial \varphi(x)}{\partial x}\right|^{2} d x \\
& -\frac{1}{l-\alpha} \int_{0}^{\tau} \int_{\alpha}^{l} x^{m} \frac{\partial u}{\partial x} \frac{\partial u}{\partial t} d x d t \\
& -\int_{0}^{\tau} \int_{\alpha}^{l} \frac{1}{l-\alpha} \frac{1}{x^{m}} \frac{\partial}{\partial x}\left(x^{m} \frac{\partial u}{\partial x}\right) \int_{\alpha}^{x} \xi^{m} \frac{\partial u(\xi, t)}{\partial t} d \xi d x d t \\
& =\frac{1}{l-\alpha} \int_{0}^{\tau} \int_{\alpha}^{l} x^{m} \frac{\partial u}{\partial x} \frac{\partial u}{\partial t} d x d t \\
& -\frac{m}{l-\alpha} \int_{0}^{\tau} \int_{\alpha}^{l} \frac{1}{x} \frac{\partial u}{\partial x} \int_{\alpha}^{x} \xi^{m} \frac{\partial u(\xi, t)}{\partial t} d \xi d x d t \\
& =\frac{1}{l-\alpha} \int_{0}^{\tau} \int_{\alpha}^{l} x^{m} \frac{\partial u}{\partial x} \frac{\partial u}{\partial t} d x d t \\
& -\frac{m}{l-\alpha} \int_{0}^{\tau} \int_{\alpha}^{l} \frac{u}{x^{2}} \int_{\alpha}^{x} \xi^{m} \frac{\partial u(\xi, t)}{\partial t} d \xi d x d t \\
& +\frac{m}{2(l-x)} \int_{\alpha}^{l} u^{2}(x, \tau) x^{m-1} d x \\
& -\frac{m}{2(l-x)} \int_{\alpha}^{l} x^{m-1} \varphi^{2}(x) d x .
\end{aligned}
$$

The formulas (14) imply the following:

$$
\begin{aligned}
-\int_{0}^{\tau} \int_{0}^{\alpha} \frac{1}{x^{m}} \frac{\partial}{\partial x}\left(x^{m} \frac{\partial u}{\partial x}\right) M \frac{\partial u}{\partial t} d x d t \\
=\frac{1}{2}\left\{\frac{1}{2} \int_{0}^{\alpha} x^{m}\left(\frac{\partial u(x, \tau)}{\partial x}\right)^{2} d x\right. \\
\quad+\int_{\alpha}^{l}\left[\frac{l-x}{l-\alpha} x^{m}\left(\frac{\partial u(x, \tau)}{\partial x}\right)^{2}+\frac{m \cdot x^{m}}{(l-\alpha)} u^{2}(x, \tau)\right] d x
\end{aligned}
$$

$$
\begin{gathered}
-\int_{0}^{\alpha} x^{m}\left|\frac{\partial \varphi(x)}{\partial x}\right|^{2} d x \\
-\int_{\alpha}^{l}\left[\frac{l-x}{l-\alpha} x^{m}\left|\frac{\partial \varphi(x)}{\partial x}\right|^{2}\right. \\
\left.\left.-\frac{m}{l-\alpha} x^{m-1} \varphi^{2}(x)\right] d x\right\} \\
-\frac{m}{l-\alpha} \int_{0}^{\tau} \int_{0}^{\alpha} \frac{u(x, t)}{x^{2}} \int_{\alpha}^{x} \xi^{m} \frac{\partial u(\xi, t)}{\partial t} d \xi d t d x
\end{gathered}
$$

Adding (13) and (15), we get

$$
\begin{gathered}
\int_{0}^{\tau} \int_{0}^{l} L u M \frac{\partial u}{\partial t} d x d t \\
=\int_{0}^{\tau} \int_{0}^{l} \psi(x) x^{m}\left|\frac{\partial u}{\partial t}\right|^{2} d x d t \\
+\frac{1}{2}\left\{\int_{0}^{l} \psi(x) x^{m}\left(\frac{\partial u(x, \tau)}{\partial x}\right)^{2} d x\right. \\
\quad+\frac{m}{(l-\alpha)} \int_{\alpha}^{l} x^{m-1} u^{2}(x, \tau) d x \\
-\int_{0}^{l} \psi(x) x^{m}\left|\frac{d \varphi}{d x}\right|^{2} d x \\
\left.+\frac{m}{(l-\alpha)} \int_{\alpha}^{l} x^{m-1} \varphi^{2}(x) d x\right\} \\
\quad-\frac{m}{(l-\alpha)} \int_{0}^{\tau} \int_{\alpha}^{l} \frac{1}{x^{m+1}}\left|\int_{\alpha}^{x} \xi_{\alpha}^{m} \frac{\partial u(\xi, t)}{\partial t}\right|^{2} d x d t
\end{gathered}
$$

where the function

$$
\psi(x)= \begin{cases}1, & 0 \leq x \leq \alpha \\ \frac{l-x}{l-\alpha}, & \alpha \leq x \leq l\end{cases}
$$

Now, it can be shown that the following inequalities hold:

$$
\begin{aligned}
& \int_{0}^{\tau} \int_{\alpha}^{l} \frac{u}{x^{2}} \int_{\alpha}^{x} \xi^{m} \frac{\partial u(\xi, t)}{\partial t} d x d t \\
& \leq \int_{0}^{\tau} \int_{\alpha}^{l} \frac{1}{x^{m+1}}\left|\int_{\alpha}^{x} \xi^{m} \frac{\partial u(\xi, t)}{\partial t}\right|^{2} d x d t \\
& \quad+\frac{1}{4 \alpha^{2}} \int_{0}^{\tau} \int_{\alpha}^{l} x^{m-1} u^{2}(x, t) d x d t,
\end{aligned}
$$




$$
\begin{gathered}
\int_{0}^{\tau} \int_{0}^{l} L u M \frac{\partial u}{\partial t} d x d t \\
\leq \frac{1}{2} \int_{0}^{\tau} \int_{0}^{l} x^{m}|L u|^{2} d x d t \\
+\frac{1}{2} \int_{0}^{\tau} \int_{0}^{l} \psi(x) x^{m}\left|\frac{\partial u}{\partial t}\right|^{2} d x d t \\
l-x \leq \psi(x) \leq 1 .
\end{gathered}
$$

The equality (16) and the inequalities (18) imply the following inequality:

$$
\begin{aligned}
\int_{0}^{\tau} \int_{0}^{l}(l-x) x^{m}\left|\frac{\partial u}{\partial t}\right|^{2} d x d t & +\int_{0}^{l}(l-x) x^{m}\left|\frac{\partial u(x, \tau)}{\partial x}\right|^{2} d x \\
& +\frac{m}{l-\alpha} \int_{\alpha}^{l} x^{m-1} u^{2}(x, \tau) d x \\
\leq & \frac{1}{2 \alpha^{2}} \frac{m}{l-\alpha} \int_{0}^{\tau} \int_{\alpha}^{l} x^{m-1} u^{2} d x d t \\
& +\int_{0}^{l} x^{m}\left|\frac{\partial \varphi}{\partial x}\right|^{2} d x \\
& +\frac{m}{l-\alpha} \int_{\alpha}^{l} x^{m-1} \varphi^{2}(x) d x \\
& +\int_{0}^{\tau} \int_{0}^{l} x^{m}(L u)^{2} d x d t .
\end{aligned}
$$

Lemma 2. Let on $[0, T]$ continuous nonnegative functions $g_{1}, g$, and $h$ be given, where $h$ is nondecreasing. Then from the inequality

$$
g_{1}(\tau)+g(\tau) \leq c \int_{0}^{\tau} g(t) d t+h(\tau)
$$

implies the inequality

$$
g_{1}(\tau)+g(\tau) \leq e^{c \tau} h(\tau)
$$

The above lemma can be proved by iteration method. We omit the details.

In order to apply the lemma, we set

$$
g(\tau)=\frac{m}{l-\alpha} \int_{\alpha}^{l} x^{m-1} u^{2}(x, \tau) d x .
$$

Let $g_{1}(\tau)$ be the first two terms in the left hand side of (19), $h(\tau)$ the last three terms in the right hand side of (19), and $c=1 / 2 \alpha^{2}$.
As a consequence of (19), we obtain the following inequality:

$$
\begin{aligned}
& \int_{0}^{\tau} \int_{0}^{l}(l-x) x^{m}\left|\frac{\partial u}{\partial t}\right|^{2} d x d t \\
& \quad+\int_{0}^{l}(l-x) x^{m}\left|\frac{\partial u(x, \tau)}{\partial x}\right|^{2} d x \\
& \quad+\frac{m}{l-\alpha} \int_{\alpha}^{l} x^{m-1} u^{2}(x, \tau) d x \leq e^{T / 2 \alpha^{2}}\|\mathscr{F}\|_{F}^{2} .
\end{aligned}
$$

Here we have used the notation (7). The right hand side of inequality (23) does not depend on $\mathscr{F}$. Consequently, in the right hand side of (23) the supremum can be taken. Thus, we get the following inequality:

$$
\begin{aligned}
& \int_{0}^{T} \int_{0}^{l}(l-x) x^{m}\left|\frac{\partial u}{\partial t}\right|^{2} d x d t \\
& +\sup _{0 \leq t \leq T}\left[\int_{0}^{l}(l-x) x^{m}\left(\frac{\partial u}{\partial x}\right)^{2} d x\right. \\
& \left.\quad+\frac{m}{l-\alpha} \int_{\alpha}^{l} x^{m-1} u^{2}(x, t) d x\right] \\
& \leq e^{T / 2 \alpha^{2}}\|\mathscr{F}\|_{F}^{2} .
\end{aligned}
$$

By (1), we get the following inequality:

$$
\begin{gathered}
\int_{0}^{T} \int_{0}^{l}(l-x) \frac{1}{x^{m}}\left(\frac{\partial}{\partial x}\left(x^{m} \frac{\partial u}{\partial x}\right)\right)^{2} d x d t \\
\leq 2 \int_{0}^{T} \int_{0}^{l}(l-x) x^{m}\left|\frac{\partial u}{\partial t}\right|^{2} d x d t \\
\quad+2 l \int_{0}^{T} \int_{0}^{l} x^{m}|f|^{2} d x d t .
\end{gathered}
$$

The inequalities (24) and (25) imply inequality (9).

\section{Conflict of Interests}

The author declares that there is no conflict of interests regarding the publication of this paper.

\section{References}

[1] A. A. Samarski1̆, "Some problems in differential equtions theory," Differentsial'nye Uravnenya, vol. 16, no. 11, pp. 1925-1935, 1980.

[2] N. B. Benouar and N. I. Yurchuk, "Mixed problem with an integral condition for parabolic equations with a Bessel operator," Differentsial'nye Uravneniya, vol. 27, no. 12, pp. 20942098, 1991.

[3] A. Bouziani and N.-E. Benouar, "Mixed problem with integral conditions for a third order parabolic equation," Kobe Journal of Mathematics, vol. 15, no. 1, pp. 47-58, 1998.

[4] A. Bouziani, "Mixed problem with boundary integral conditions for a certain parabolic equation," Journal of Applied Mathematics and Stochastic Analysis, vol. 9, no. 3, pp. 323-330, 1996. 
[5] J. R. Cannon, The One-Dimensional Heat Equation, vol. 23 of Encyclopedia of Mathematics and its Applications, AddisonWesley, Menlo Park, Calif, USA, 1984.

[6] J. R. Cannon, "The solution of the heat equation subject to the specification of energy," Quarterly of Applied Mathematics, vol. 21, no. 2, pp. 155-160, 1963.

[7] J. R. Cannon and J. van der Hoek, "The existence of and a continuous dependence result for the solution of the heat equation subject to the specification of energy," Bollettino dell'Unione Matematica Italiana, supplement 1, pp. 253-282, 1981.

[8] N. I. Ionkin, "Solution of a certain boundary value problem of the theory of heat conduction with a nonclassical boundary condition," Differentsial'nye Uravneniya, vol. 13, no. 2, pp. 294304, 1977.

[9] N. I. Ionkin and E. I. Moiceev, "Solution of a certain boundary value problem of the theory of heat conduction with a nonclassical boundary condition," Differentsial'nye Uravneniya, vol. 13, no. 2, pp. 294-304, 1977.

[10] N. I. Kamynin, "A boundary value problem in the theory of heat conduction with non-classical boundary condition," Žurnal Vyčislitel' noŭ Matematiki i Matematičeskoŭ Fiziki, vol. 4, no. 6, pp. 1006-1024, 1964.

[11] N. I. Yurchuk, "Mixed problem with an integral condition for some parabolic equations," Differentsial'nye Uravnenya, vol. 22, no. 12, pp. 2117-2126, 1986.

[12] N. I. Yurchuk, "Solvability of boundary value problems for certain operator-differential equations," Differentsial'nye Uravnenya, vol. 13, no. 4, pp. 626-636, 1977.

[13] A. Bouziani, "On the solvability of a class of singular parabolic equations with nonlocal boundary conditions in nonclassical function spaces," International Journal of Mathematics and Mathematical Sciences, vol. 30, no. 7, pp. 435-447, 2002.

[14] L. Byszewski, "Existence and uniqueness of solutions of nonlocal problems for hyperbolic equation $u_{x t}=F\left(x, t, u, u_{x}\right)$," Journal of Applied Mathematics and Stochastic Analysis, vol. 3, no. 3, pp. 163-168, 1990.

[15] L. Byszewski, "Theorem about existence and uniqueness of continuous solution of nonlocal problem for nonlinear hyperbolic equation," Applicable Analysis, vol. 40, no. 2-3, pp. 173-180, 1991.

[16] L. Byszewski, Differential and Functional-Differential Problems Together with Nonlocal Conditions, Monograph 184, Cracow University of Technology, Cracow, Poland, 1995.

[17] È. A. Gasymov, "Mixed problems for conjugation of parabolic systems of different orders with nonlocal boundary conditions," Differentsial'nye Uravneniya, vol. 26, no. 8, pp. 1003-1012, 1990.

[18] B. Lazhar, "Parabolic equations with nonlocal conditions," Applied Mathematical Sciences, vol. 1, no. 21, pp. 1041-1048, 2007.

[19] M. Said and L. Nadia, "On a nonlocal singular mixed evolution problem," Matematichki Vesnik, vol. 53, no. 3-4, pp. 79-89, 2001.

[20] Z. D. Moussa, K. Tcharie, and N. I. Yurchuk, "Continuous dependence of solutions to mixed boundary value problems for a parabolic equation," Electronic Journal of Differential Equations, vol. 2008, no. 17, pp. 1-10, 2008. 


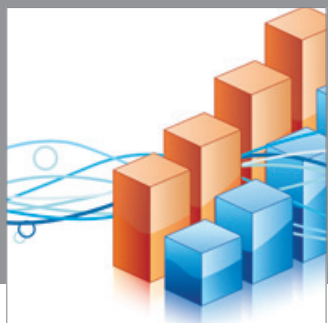

Advances in

Operations Research

mansans

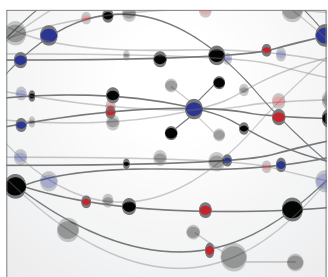

The Scientific World Journal
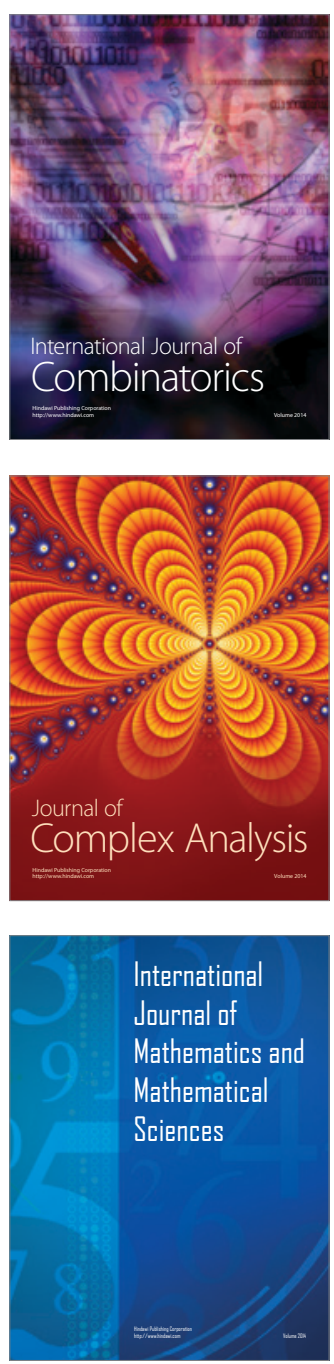
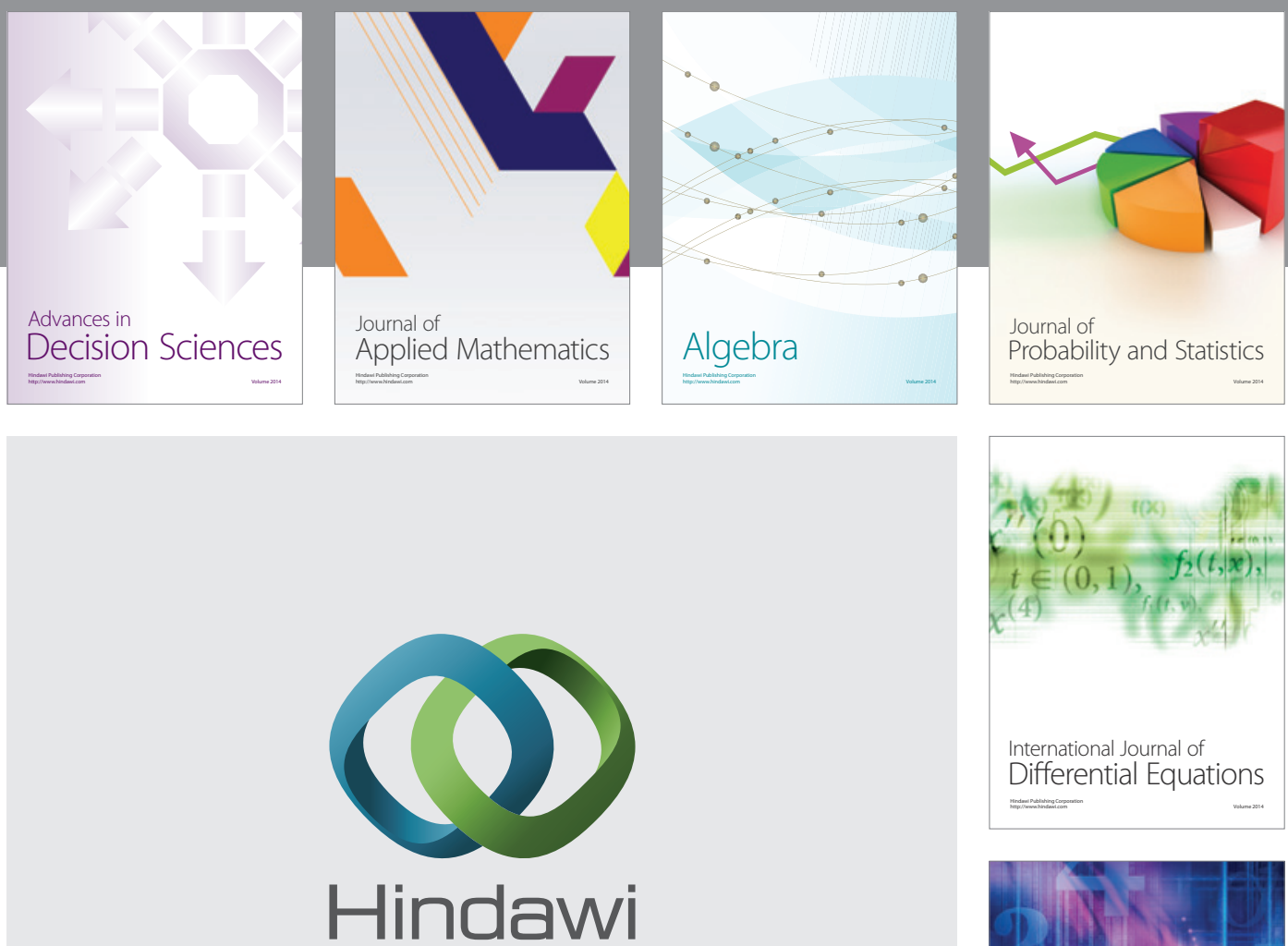

Submit your manuscripts at http://www.hindawi.com
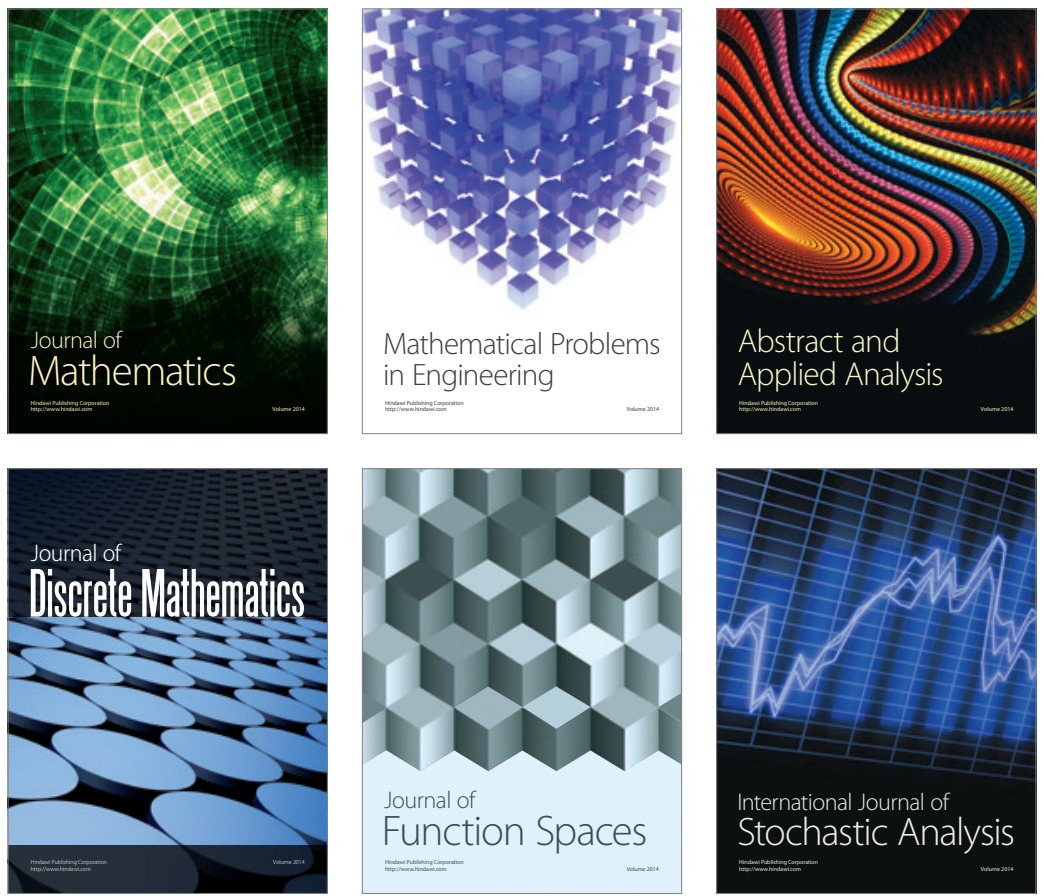

Journal of

Function Spaces

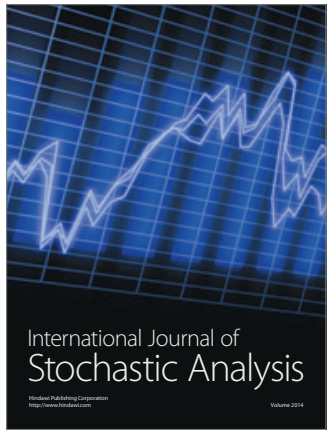

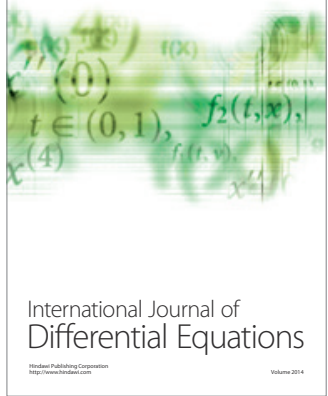
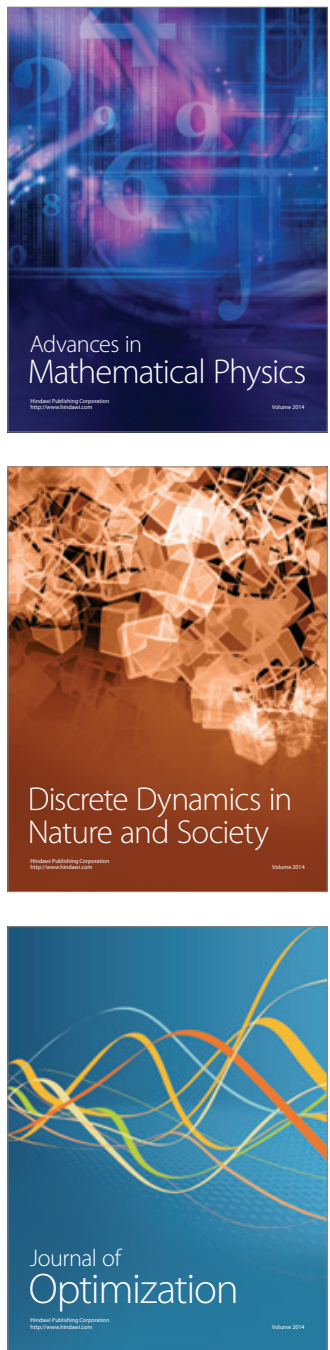